\title{
Association between vitamin $D$ insufficiency and adverse pregnancy outcome: global comparisons
}

This article was published in the following Dove Press journal:

International Journal of Women's Health

3 September 2013

Number of times this article has been viewed

\author{
Abdulbari Bener ${ }^{1,2}$ \\ Abdulla OAA Al-Hamaq ${ }^{3}$ \\ Najah M Saleh ${ }^{4}$ \\ 'Department of Medical Statistics \\ and Epidemiology, Hamad General \\ Hospital, Doha, Qatar; ${ }^{2}$ Institute \\ of Population Health, University of \\ Manchester, Manchester, UK; ${ }^{3} \mathrm{Q} a t a r$ \\ Diabetic Association, Doha, Qatar; \\ ${ }^{4}$ Department of Obstetrics and \\ Gynecology, Women's Hospital, \\ Doha, Qatar
}

Background: Vitamin D insufficiency has been associated with a number of adverse pregnancy outcomes, and has been recognized as a public health concern.

Aim: The objective of this study was to determine the impact of vitamin D deficiency on maternal complications like gestational diabetes mellitus (GDM), anemia, iron deficiency, and preeclampsia among pregnant women.

Subjects and methods: This was a cohort study undertaken at antenatal clinics at the Women's Hospital of Hamad Medical Corporation in Doha. A total of 2,487 Arab pregnant women above 24 weeks' gestation with any maternal complication were approached, and 1,873 women $(75.3 \%)$ consented to participate in the study. Data on sociodemographic and clinical characteristics by interview and biochemistry parameters were retrieved from medical records. Multivariate logistic regression analysis was performed to determine the associated risk factors.

Results: Of the studied pregnant women, nearly half of them had vitamin D deficiency (48.4\%). Younger women below 30 years old $(43.2 \%, P=0.032)$, housewives $(65.3 \%, P=0.008)$, and those on low monthly household incomes (QR5,000-9,999) $(49.2 \%, P=0.03)$ were significantly more likely to have lower vitamin D compared with those who had sufficient vitamin D levels. Exposure to sunlight $(63.4 \%, P=0.05)$, daily physical activity $(64.4 \%, P=0.05)$, and vitamin D supplement intake $(89.7 \%, P<0.001)$ were significantly lower in deficient pregnant women. In the study sample of pregnant women, $13.9 \%$ had GDM, $11.5 \%$ had anemia, $8.6 \%$ had iron deficiency, and $6.9 \%$ had preeclampsia. Severe vitamin D deficiency was significantly higher in pregnant women with GDM (16.5\% vs $11 \%)$, anemia (17.1\% vs $11 \%)$, iron deficiency $(18.5 \%$ vs $11.2 \%$ ), and preeclampsia (19.8\% vs $11.4 \%$ ) when compared to the uncomplicated group. Socioeconomic status was low in pregnant women with complications like GDM, anemia, iron deficiency, and pre-eclampsia. Pregnancy complications like GDM (52.7\%), anemia (53.2\%), iron deficiency (55.6\%), and preeclampsia (51.9\%) were higher in Qataris. Also, GDM (66.2\%), anemia (66.2\%), iron deficiency $(68.5 \%)$, and preeclampsia $(58.1 \%)$ were observed more commonly among housewives compared to working women. Obesity was significantly more common in pregnant women with GDM (41.5\%) and preeclampsia (41.1\%).

Conclusion: The study findings revealed that maternal vitamin D deficiency in pregnancy is significantly associated with elevated risk for GDM, anemia, and preeclampsia. The risk of vitamin D deficiency was higher in Qataris, housewives and those with low monthly household income.

Keywords: pregnant women, vitamin D deficiency, GDM, anemia, iron deficiency, preeclampsia

Department of Medical Statistics and Epidemiology, Hamad General Hospital, Hamad Medical Corporation, PO Box 3050, Doha, Qatar Tel +974 $44393765 / 6$

Fax +974 44393769

Email abener@hmc.org.qa

\section{Introduction}

Complications of pregnancy and childbirth are the leading causes of disability and death among women of reproductive age in developing countries, accounting for at 
least $18 \%$ of the global burden of disease in this age-group. ${ }^{1}$ Similarly, the pattern of leading causes of maternal death and disability are closely linked to high prevalence of vitamin D deficiency, poor maternal health during pregnancy, inadequate care during delivery, and lack of newborn care. ${ }^{1}$ Every year, almost 8 million stillbirths and early neonatal deaths occur. In addition to maternal deaths, more than 50 million women experience maternal health problems annually. ${ }^{2}$ In underdeveloped countries, those high vitamin D deficiency and maternal complications often pose an immediate financial burden on women and their households.

Vitamin D deficiency during pregnancy is common in many parts of the world, ${ }^{3}$ and there is a very strong relationship between vitamin deficiency $\mathrm{D}$ and multiple potential adverse pregnancy outcomes. ${ }^{4-7}$ However, the role and metabolism of vitamin D in the pregnant state is not well known or understood. ${ }^{5,8-10}$ Despite the reported high prevalence of deficiency and the possible consequences, the desired optimal level needed for pregnant women in their body and the amount of vitamin $\mathrm{D}$ intake required to maintain adequate levels is not very well documented. ${ }^{3-12}$

The vitamin D stores in the infant start with transplacental transfer of $25(\mathrm{OH}) \mathrm{D}$ in early pregnancy from mother to fetus. ${ }^{5,13}$ It is very obvious that maintaining optimum vitamin D nutrition during pregnancy is essential for prevention of hypovitaminosis D in the fetus and vitamin D deficiency at birth and in early infancy. ${ }^{13}$

An estimated 1.6 billion people are anemic worldwide, and anemia is common during pregnancy. Approximately 50\% of pregnant women have anemia (hemoglobin $<110 \mathrm{~g} / \mathrm{L}$ ) in resource-limited settings, compared with $12 \%-25 \%$ in developed regions. ${ }^{14}$ Anemia is a global public health problem affecting both developing and developed countries, with major consequences for human health as well as social and economic development. It occurs at all stages of the life cycle, but is more prevalent in pregnant women and young children. In 2002, iron-deficiency anemia (IDA) was considered to be among the most important contributing factors to the global burden of disease. ${ }^{14}$ Anemia is the result of a wide variety of causes that can be isolated, but more often coexist. Globally, the most significant contributor to the onset of anemia is iron deficiency, so "IDA" and "anemia" are often used synonymously, and the prevalence of anemia has often been used as a proxy for IDA. It is generally assumed that $50 \%$ of the cases of anemia are due to iron deficiency, ${ }^{14}$ but the proportion may vary among population groups and in different areas according to the local conditions. The main risk factors for IDA include a low intake of iron, poor absorption of iron from diets high in phytate or phenolic compounds, and period of life when iron requirements are especially high (ie, growth and pregnancy).

In recent years, the prevalence of vitamin $\mathrm{D}$ deficiency has increased, and the incidence of low vitamin D status has risen in the developing world and in the UK and other developed countries. ${ }^{11,15-17}$ There is a high prevalence of vitamin D deficiency in pregnant women from non-Western countries in Northern Europe, and vitamin D deficiency during pregnancy is an ongoing epidemic. ${ }^{18}$

At present, there is not enough evidence to evaluate the effectiveness of vitamin D in pregnancy, and therefore vitamin D supplementation is not routinely offered to all pregnant women in Qatar. Many studies have investigated vitamin D deficiency in pregnancy in different ethnic minority groups and the method of supplementation. ${ }^{19}$ There is evidence that an immediate dose is as effective as a daily dose, with no adverse effects. ${ }^{13-18}$

The objective of the study was to determine the impact of vitamin D deficiency on gestational diabetes mellitus (GDM), anemia, iron deficiency, and preeclampsia medical conditions among pregnant women.

\section{Subjects and methods}

This was a cohort study conducted among pregnant Arab women in the third trimester from January 2011 to December 2011. The study was based on the logbook of the Women's Hospital, which registers all pregnant women visiting antenatal clinics at the Women's Hospital, Hamad Medical Corporation. The research assistants screened the logbook of the Women's Hospital during the study period and prepared a list of 2,487 pregnant Arab women above 24 weeks of gestation with any maternal complication. All the eligible women on the prepared list $(n=2,487)$ were approached, and consent was sought. Only 1,873 pregnant women (75.3\%) participated in the study (refer to Figure 1). Nearly 25\% of the approached subjects were excluded from the study, because some of them had incomplete medical records as well as incomplete questionnaires. A few of them did not give consent due to their busy schedule and lack of time to fill the questionnaire. Moreover, our study sample included 1,873 pregnant women, which is approximately $10 \%$ of total mothers that delivered in the year 2011. Each participant was provided with brief information about the study and was assured of strict confidentiality.

The study was approved by the Research Ethics Committee of Hamad Medical Corporation and by Institutional Review Board of Weill Cornell Medical College. All human studies 


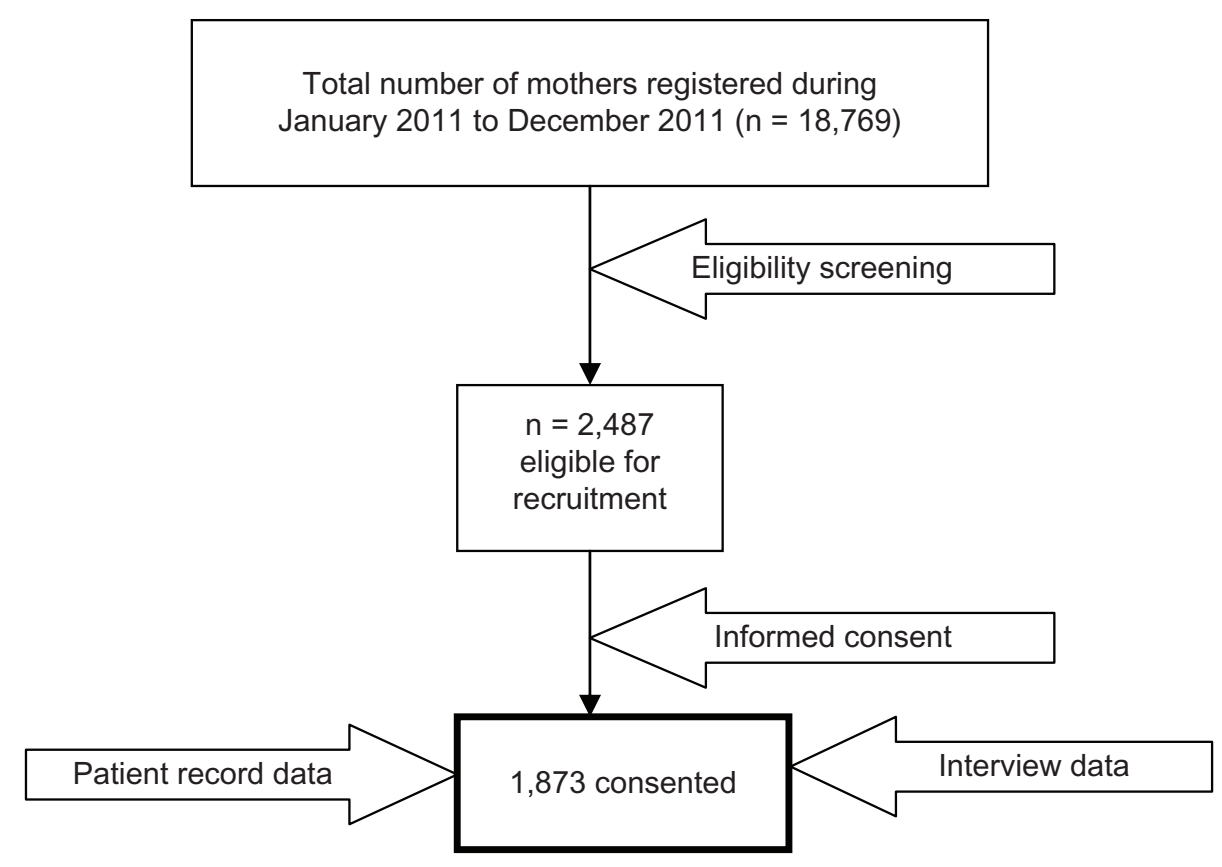

Figure I Flow diagram of representative sample studied $(\mathrm{n}=1,873)$.

are approved by the Research Ethics Committee, and therefore are performed in accordance with the ethical standards laid down in the 1964 Declaration of Helsinki. All the persons who agreed to participate in this study gave their informed consent prior to their inclusion in the study.

Data were collected through face-to-face interviews by qualified nurses using a validated questionnaire in the local language. The questionnaire covered sociodemographic characteristics of the pregnant women, family and medical histories, anthropometric and obstetric characteristics, and pregnancy outcomes. Maternal medical records were reviewed to collect detailed medical and clinical information. The questionnaire was pilot-tested on 100 randomly selected pregnant women for validity. The investigators made necessary corrections and modifications after considering minor differences and discrepancies that were found during the pilot study. Data on maternal complications of each patient were retrieved from the medical record file. In addition, follow-up data on pregnancy and neonatal outcome of each woman were obtained from the labor-room register immediately after delivery (Figure 1).

\section{Laboratory investigations}

Blood collection and serum measurements of vitamin D

Serum concentrations of vitamin D metabolites were determined by radioimmunoassay kits from Immunodiagnostic Systems Ltd, Boldon, UK. A trained phlebotomist collected venous blood samples, separated serum, and stored at $-70^{\circ} \mathrm{C}$ until analysis. Serum 25-hydroxyvitamin D (25[OH]D), a vitamin D metabolite, was measured using a commercially available kit (DiaSorin, Saluggia, Italy). The treated samples were then assayed using competitive-binding radioimmunoassay. Subjects were classified into four categories: (1) severe vitamin D deficiency, $25(\mathrm{OH}) \mathrm{D}<10 \mathrm{ng} / \mathrm{mL}$; (2) moderate deficiency, 25(OH)D 10-19 ng/mL; (3) mild deficiency, 25(OH)D 20-29 ng/mL; and (4) normal/optimal level, between 30 and $80 \mathrm{ng} / \mathrm{mL},{ }^{13,15}$ which is consistent with other studies. ${ }^{18,19}$ Other baseline biochemical parameters measured from the serum included vitamin $\mathrm{D}$, calcium, albumin, bilirubin, magnesium, calcium, cholesterol, urea, triglyceride, and phosphorus and parathyroid hormone levels. On the basis of previous recommendations, ${ }^{19-21}$ serum levels of these biochemical parameters were determined according to standard laboratory procedures. Furthermore, during the screening period, each patient provided a complete history, and a comprehensive examination was performed.

Preeclampsia was defined as gestational hypertension and proteinuria and return of all abnormalities to normal by 12 weeks postpartum. Gestational hypertension was defined according to $\mathrm{WHO}^{20}$ criteria as systolic blood pressure $\geq 140 \mathrm{mmHg}$ and/or diastolic blood pressure $\geq 90 \mathrm{mmHg}$ and/or higher for the first time after 20 weeks' gestation. According to the recommendations from the WHO Expert Committee, ${ }^{21}$ pregnant women were screened at 24-28 weeks for GDM, which was defined according to 
fasting venous blood glucose concentration $\geq 7.0 \mathrm{mmol} / \mathrm{L}$ and/or 2 hours post-oral glucose tolerance test venous blood glucose concentration $\geq 11.1 \mathrm{mmol} / \mathrm{L}$. The $\mathrm{WHO}^{22}$ tabulation adopts the international definition for anemia for pregnant women of $<110 \mathrm{~g} / \mathrm{L}$. Anemia was defined as a hemoglobin level of $<11 \mathrm{~g} / \mathrm{dL}$ for women.

Data were analyzed using SPSS version 20 (IBM, Armonk, NY, USA). Student's $t$-test was used to ascertain the significance of differences between mean values of two continuous variables and confirmed by the nonparametric Mann-Whitney test. Chi-squares analysis was performed to test for differences in proportions of categorical variables between two or more groups. In $2 \times 2$ tables, Fisher's exact test (two-tailed) replaced the chi-squared test if the assumptions underlying chi-squared were violated, namely in cases of small sample size and where the expected frequency was less than five in any of the cells. Pearson's correlation coefficient was used to evaluate the strength association between two continuous variables. All statistical tests were two-sided, and $P<0.05$ was considered statistically significant.

\section{Results}

Table 1 shows the sociodemographic characteristics and lifestyle habits of the studied pregnant women according to vitamin D status. Nearly half of the studied pregnant women had vitamin D deficiency (48.4\%). Of women at high risk of vitamin D deficiency, younger women below 30 years old (43.2\%, $P=0.032)$, housewives (65.3\%, $P=0.008)$, and those with low monthly household income (QR5,000-9,999) (49.2\%, $P=0.03$ ) were significantly more likely to have lower vitamin $\mathrm{D}$ compared with those of higher socioeconomic status. In terms of lifestyle habits, vitamin D deficiency was higher in pregnant women who had less exposure to sun $(63.4 \%$ vs $59 \%, P=0.05)$ and no daily physical activity $(64.4 \%$ vs $59.0 \%, P=0.05)$ compared to the vitamin D-sufficient group. Vitamin D supplement intake was significantly lower in vitamin D-deficient pregnant women (89.7\%, $P<0.001)$.

Table 2 shows the sociodemographic characteristics of studied pregnant women with the complications of GDM, anemia, iron deficiency, and preeclampsia. GDM (52.7\% vs $47.3 \%$ ), anemia (53.2\% vs $46.8 \%$ ), iron deficiency $(55.6 \%$ vs $44.4 \%)$, and preeclampsia (51.9\% vs $48.1 \%$ ) were more frequent among pregnant Qatari women than in pregnant non-Qatari women. The pregnancy complications of GDM $(52.7 \%)$, anemia $(53.2 \%)$, iron deficiency $(55.6 \%)$, and preeclampsia $(51.9 \%)$ were higher in Qataris. Housewives had higher pregnancy complications - GDM (66.2\%),
Table I Sociodemographic characteristics and lifestyle habits of the studied pregnant women according to vitamin $D$ status $(n=1,873)$

\begin{tabular}{|c|c|c|c|c|}
\hline \multirow[t]{2}{*}{ Variables } & \multirow{2}{*}{$\begin{array}{l}\text { Total, } \\
\text { n (\%) }\end{array}$} & \multicolumn{3}{|c|}{ Vitamin D status } \\
\hline & & $\begin{array}{l}\text { Deficient } \\
(n=907), \\
n(\%)\end{array}$ & $\begin{array}{l}\text { Sufficient } \\
(n=966), \\
n(\%)\end{array}$ & $P$-value \\
\hline \multicolumn{5}{|l|}{ Nationality } \\
\hline Qatari & $907(48.42)$ & $426(47.0)$ & $481(49.8)$ & 0.22 \\
\hline Non-Qatari & $966(51.58)$ & $481(53.0)$ & $485(50.2)$ & \\
\hline \multicolumn{5}{|l|}{ Age-group } \\
\hline$<30$ years & $746(39.83)$ & $392(43.2)$ & $354(36.6)$ & 0.03 \\
\hline 30-34 years & $457(24.40)$ & $212(23.4)$ & $245(25.4)$ & \\
\hline $35-39$ years & $383(20.45)$ & $170(18.7)$ & $213(22.0)$ & \\
\hline $40-45$ years & $287(15.32)$ & $133(14.7)$ & $154(15.9)$ & \\
\hline \multicolumn{5}{|c|}{ Body mass index group $\left(\mathrm{kg} / \mathrm{m}^{2}\right)$} \\
\hline $20-24.99$ & $854(45.6)$ & $4 I I(45.3)$ & $443(45.9)$ & 0.93 \\
\hline \multicolumn{5}{|l|}{ (normal) } \\
\hline $25-30$ & $539(28.8)$ & $260(28.7)$ & $279(28.9)$ & \\
\hline \multicolumn{5}{|l|}{ (overweight) } \\
\hline$>30$ (obese) & $480(25.6)$ & $236(26.0)$ & $244(25.3)$ & \\
\hline \multicolumn{5}{|c|}{ Education level } \\
\hline Illiterate & $118(6.30)$ & $65(7.2)$ & $53(5.5)$ & 0.48 \\
\hline Primary & $167(8.92)$ & $77(8.5)$ & $90(9.3)$ & \\
\hline Intermediate & $172(9.18)$ & $89(9.8)$ & $83(8.6)$ & \\
\hline Secondary & $631(33.69)$ & $303(33.4)$ & $328(34.0)$ & \\
\hline University & $785(41.91)$ & $373(4 I .1)$ & $412(42.7)$ & \\
\hline \multicolumn{5}{|l|}{ Occupation } \\
\hline Housewife & $\mathrm{I}, \mathrm{I} 64(62.15)$ & $592(65.3)$ & $572(59.2)$ & 0.01 \\
\hline Sedentary/ & $495(26.43)$ & $225(24.8)$ & $270(28.0)$ & \\
\hline \multicolumn{5}{|l|}{ professional } \\
\hline Manual & $128(6.83)$ & $61(6.7)$ & $67(6.9)$ & \\
\hline Business man & $53(2.83)$ & $21(2.3)$ & $32(3.3)$ & \\
\hline Army/police & $33(1.76)$ & $8(0.9)$ & $25(2.6)$ & \\
\hline \multicolumn{5}{|c|}{ Monthly household income (QR)* } \\
\hline $5000-9999$ & $885(47.25)$ & $446(49.2)$ & $439(45.4)$ & 0.03 \\
\hline $10,000-14,999$ & $393(20.98)$ & $198(21.8)$ & $195(20.2)$ & \\
\hline $15,000-20,000$ & $403(21.52)$ & $169(18.6)$ & $234(24.2)$ & \\
\hline$>20,000$ & $192(10.25)$ & $94(10.4)$ & $98(10.1)$ & \\
\hline \multicolumn{5}{|c|}{ Place of residence } \\
\hline Urban & $1,612(86.07)$ & $787(86.8)$ & $825(85.4)$ & 0.36 \\
\hline Rural & $26 \mid(13.93)$ & $120(13.2)$ & $14 \mid(14.6)$ & \\
\hline \multicolumn{5}{|c|}{ Consanguineous marriages } \\
\hline \multicolumn{5}{|l|}{ First cousin } \\
\hline Yes & $803(42.87)$ & $399(44.0)$ & $404(41.8)$ & 0.34 \\
\hline No & $1,070(57.13)$ & $508(56.0)$ & $562(58.2)$ & \\
\hline \multicolumn{5}{|l|}{ Second cousin } \\
\hline Yes & $629(33.58)$ & $319(35.2)$ & $310(32.1)$ & 0.16 \\
\hline No & $1,244(66.42)$ & $588(64.8)$ & $656(67.9)$ & \\
\hline \multicolumn{5}{|c|}{ Smoking cigarettes/water pipe } \\
\hline Yes & $170(9.08)$ & $88(9.7)$ & $82(8.5)$ & 0.36 \\
\hline No & I,703 (90.92) & $819(90.3)$ & $884(91.5)$ & \\
\hline \multicolumn{5}{|c|}{ Exposure to sun } \\
\hline Yes & 728 (38.9) & $332(36.6)$ & $396(4 I)$ & 0.05 \\
\hline No & $1,145(61.1)$ & $575(63.4)$ & $570(59)$ & \\
\hline \multicolumn{5}{|c|}{ Daily physical activity } \\
\hline Yes & $719(38.76)$ & $323(35.6)$ & $396(41.0)$ & 0.05 \\
\hline No & $\mathrm{I}, 154(6 \mathrm{I} .24)$ & $584(64.4)$ & $570(59.0)$ & \\
\hline Supplement & vitamin D & & & \\
\hline Yes & $609(32.5 \mathrm{I})$ & $93(10.3)$ & $516(53.4)$ & $<0.001$ \\
\hline No & I,264 (67.49) & $814(89.7)$ & $450(46.6)$ & \\
\hline
\end{tabular}

Notes: *US\$I = QR3.65. 
Table 2 Sociodemographic characteristics of pregnant women with complications like gestational diabetes mellitus (GDM), anemia, iron deficiency, and preeclampsia $(n=1,873)$

\begin{tabular}{|c|c|c|c|c|}
\hline Variable & $\begin{array}{l}\text { GDM } \\
(n=260) \\
n(\%)\end{array}$ & $\begin{array}{l}\text { Anemia } \\
(n=2 \mid 6), \\
n(\%)\end{array}$ & $\begin{array}{l}\text { Iron } \\
\text { deficiency } \\
(n=162), \\
n(\%)\end{array}$ & $\begin{array}{l}\text { Preeclampsia } \\
(n=129), \\
n(\%)\end{array}$ \\
\hline \multicolumn{5}{|l|}{ Nationality } \\
\hline Qatari & I 37 (52.7) & $115(53.2)$ & $90(55.6)$ & $67(51.9)$ \\
\hline Non-Qatari & $123(47.3)$ & $101(46.8)$ & $72(44.4)$ & $62(48.1)$ \\
\hline \multicolumn{5}{|l|}{ Age-group } \\
\hline$<30$ years & $116(44.6)$ & $108(50.0)^{*}$ & $73(45.1)$ & $52(40.3)$ \\
\hline $30-34$ years & $50(19.2)$ & $45(20.8)$ & $35(21.6)$ & $30(23.3)$ \\
\hline $35-39$ years & $54(20.8)$ & $30(13.9)$ & $36(22.2)$ & $25(19.4)$ \\
\hline $40-45$ years & $40(15.4)$ & $33(15.3)$ & $18(11.1)$ & $22(17.1)$ \\
\hline \multicolumn{5}{|l|}{ Education } \\
\hline Illiterate & $19(7.3)$ & I 8 (8.3) & I3 (8.0) & $8(6.2)$ \\
\hline Primary & $27(10.4)$ & $15(6.9)$ & $16(9.9)$ & $7(5.4)$ \\
\hline Intermediate & $24(9.2)$ & $23(10.6)$ & $15(9.3)$ & $8(6.2)$ \\
\hline Secondary & $79(30.4)$ & $78(36.1)$ & $45(27.8)$ & $49(38.0)$ \\
\hline $\begin{array}{l}\text { College/ } \\
\text { university }\end{array}$ & III (42.7) & $82(38.0)$ & $73(45.1)$ & $57(44.2)$ \\
\hline \multicolumn{5}{|l|}{ Occupation } \\
\hline Housewife & $172(66.2)$ & $143(66.2)$ & III (68.5) & $75(58.1)$ \\
\hline Sedentary/ & $57(21.9)$ & $51(23.6)$ & $40(24.7)$ & $33(25.6)$ \\
\hline $\begin{array}{l}\text { professional } \\
\text { Clerk }\end{array}$ & $31(11.9)$ & $22(10.2)$ & II (6.8) & $21(16.3)$ \\
\hline \multicolumn{5}{|c|}{ Monthly income (QR) } \\
\hline$<10,000$ & $|3|(50.4)$ & $100(46.3)$ & $84(51.9)$ & $56(43.4)$ \\
\hline $10,000-14,999$ & $53(20.4)$ & $49(22.7)$ & $29(17.9)$ & $30(23.3)$ \\
\hline $15,000-20,000$ & $58(22.3)$ & $46(21.3)$ & $37(22.8)$ & $26(20.2)$ \\
\hline$>20,000$ & $18(6.9)$ & $21(9.7)$ & $12(7.4)$ & $17(13.2)$ \\
\hline \multicolumn{5}{|c|}{ Place of residence } \\
\hline Urban & $233(89.6)$ & $190(88.0)$ & $135(83.3)$ & II 8 (9l.5) \\
\hline Rural & $27(10.4)$ & $26(12.0)$ & $27(16.7)$ & II (8.5) \\
\hline \multicolumn{5}{|c|}{ Consanguineous marriages } \\
\hline \multicolumn{5}{|l|}{ First cousin } \\
\hline Yes & $129(49.6)^{*}$ & $113(52.3)^{*}$ & $78(48.1)^{*}$ & $57(44.2)^{*}$ \\
\hline No & $|3|(50.4)$ & $103(47.7)$ & $84(51.9)$ & $72(55.8)$ \\
\hline \multicolumn{5}{|l|}{ Second cousin } \\
\hline Yes & $95(36.5)^{*}$ & $75(34.7)^{*}$ & $52(32.1)^{*}$ & $40(31)^{*}$ \\
\hline No & $165(63.5)$ & $|4|(65.3)$ & $110(67.9)$ & $89(69)$ \\
\hline \multicolumn{5}{|c|}{ Smoking cigarettes/water pipe } \\
\hline Yes & $37(\mid 4.2)^{*}$ & $32(14.8)^{*}$ & $26(16.0)^{*}$ & $20(15.5)^{*}$ \\
\hline No & $223(85.8)$ & $184(85.2)$ & $136(84.0)$ & $108(83.7)$ \\
\hline
\end{tabular}

Notes: $* P<0.05$ when compared with uncomplicated group; + US $\$ I=Q R 3.65$.

anemia (66.2\%), iron deficiency (68.5\%), and preeclampsia (58.1\%) - compared to working women. Also, the pregnant women with low monthly household income had a higher frequency of GDM (50.4\%), anemia (46.3\%), iron deficiency $(51.9 \%)$, and preeclampsia (43.4\%).

Table 3 shows the serum level of vitamin D among pregnant women with pregnancy complications. Severe deficiency was significantly higher in pregnant women with GDM ( $16.5 \%$ vs $11 \%)$, anemia ( $17.1 \%$ vs $11 \%)$, iron deficiency (18.5\% vs $11.2 \%)$, and preeclampsia (19.8\% vs $11.4 \%$ ) when compared to the uncomplicated group. Also, moderate vitamin deficiency was significantly higher in pregnant women with GDM (38.1\% vs $36.4 \%)$ and anemia $(37.5 \%$ vs $36 \%$ ) than in the uncomplicated group.

Table 4 shows the maternal and obstetric characteristics of pregnant women with pregnancy complications: GDM, anemia, iron deficiency, and preeclampsia. Obesity was higher among pregnant women with GDM (41.5\%) and preeclampsia (41.1\%). GDM (39.6\%), anemia (45.8\%), iron deficiency (40.1\%), and preeclampsia (41.1\%) were observed more in women, with four to six parity groups.

Table 5 shows the predictors for GDM, anemia, iron deficiency, and preeclampsia in studied pregnant women using multivariate logistic regression analysis. Vitamin D deficiency was a significant contributor for GDM (odds ratio $[\mathrm{OR}] 1.38$, confidence interval $[\mathrm{CI}] 1.05-1.82 ; P=0.019)$ and anemia (OR 1.87, CI 1.32-2.63; $P<0.001$ ). Previous low birth weight (OR 1.75, CI 1.20-256; $P=0.003$ ) and consanguinity (second cousin) (OR 1.64, CI 1.22-2.20; $P=0.001$ ) were the second major contributor for GDM and anemia. Vitamin D deficiency appeared as a significant contributor for iron deficiency (OR 1.84, CI 1.13-3.32; $P=0.020)$ and preeclampsia (OR 1.75, CI 1.16-2.58; $P=0.010)$ in pregnant women.

Table 6 shows the global variations and comparison in vitamin D status during pregnancy. Low vitamin D intake was higher in the US $(24.8 \%),{ }^{40}$ the People's Republic of China $(24.2 \%),{ }^{38}$ Canada (20.7\%), ${ }^{36}$ and Australia $(21 \%),{ }^{10}$ which is in line with the present study rate $(17.8 \%)$.

\section{Discussion}

Vitamin D deficiency has been associated with several adverse health outcomes, including pregnancy outcomes, and is a public health issue worldwide. The present study demonstrated a greater risk of maternal complications in pregnant women with vitamin $\mathrm{D}$ deficiency compared with those with normal vitamin D levels. An increased risk of adverse pregnancy outcomes, such as GDM, anemia, iron deficiency and preeclampsia, was noted in the study sample of pregnant women, which is similar to the results observed by Kaludjerovic and Vieth. ${ }^{22}$ The mean serum levels of vitamin D in pregnant women with GDM (17.78 \pm 7.7$)$, anemia (17.65 \pm 7.8$)$, iron deficiency (16.01 \pm 7.4$)$, and preeclampsia $(18.01 \pm 8.6)$ revealed vitamin $D$ insufficiency in pregnant women. It was reported ${ }^{23-24}$ that vitamin D status in women with risk factors of maternal complications like GDM, anemia, and preeclampsia was 
Table 3 Serum levels of vitamin D among pregnant women according to the status of pregnancy complications

\begin{tabular}{|c|c|c|c|c|c|c|c|c|}
\hline \multirow[t]{2}{*}{$\begin{array}{l}\text { Serum level of } \\
\text { vitamin D }\end{array}$} & \multicolumn{2}{|c|}{$\begin{array}{l}\text { Gestational diabetes } \\
\text { mellitus }\end{array}$} & \multicolumn{2}{|l|}{ Anemia } & \multicolumn{2}{|c|}{ Iron deficiency } & \multicolumn{2}{|c|}{ Preeclampsia } \\
\hline & $\begin{array}{l}\text { Yes } \\
n=260\end{array}$ & $\begin{array}{l}\text { No } \\
n=1,6 \mid 3\end{array}$ & $\begin{array}{l}\text { Yes } \\
n=216\end{array}$ & $\begin{array}{l}\text { No } \\
n=1,657\end{array}$ & $\begin{array}{l}\text { Yes } \\
n=162\end{array}$ & $\begin{array}{l}\text { No } \\
n=|, 7| \mid I\end{array}$ & $\begin{array}{l}\text { Yes } \\
n=129\end{array}$ & $\begin{array}{l}\text { No } \\
n=I, 744\end{array}$ \\
\hline \multicolumn{9}{|l|}{ Severe deficiency } \\
\hline $25(\mathrm{OH}) \mathrm{D}<10 \mathrm{ng} / \mathrm{mL}$ & $43(16.5)$ & $178(\mid 1.0)$ & $37(17.1)$ & $184(\mathrm{II})$ & $30(18.5)$ & $191(11.2)$ & $21(19.8)$ & $198(11.4)$ \\
\hline \multicolumn{9}{|l|}{ Moderate deficiency } \\
\hline $25(\mathrm{OH}) \mathrm{D} 10-19 \mathrm{ng} / \mathrm{mL}$ & $99(38.1)$ & $587(36.4)$ & $81(37.5)$ & $596(36)$ & $49(30.2)$ & $637(37.2)$ & $31(29.2)$ & $647(37.1)$ \\
\hline \multicolumn{9}{|l|}{ Mild deficiency } \\
\hline $25(\mathrm{OH}) \mathrm{D} 20-29 \mathrm{ng} / \mathrm{mL}$ & $72(27.7)$ & $616(38.2)$ & $70(32.4)$ & $618(37.3)$ & $57(36.4)$ & $629(36.8)$ & $50(38.8)$ & $638(36.6)$ \\
\hline \multicolumn{9}{|l|}{ Optimal } \\
\hline $25(\mathrm{OH}) \mathrm{D} 30-80 \mathrm{ng} / \mathrm{mL}$ & $46(17.7)$ & $232(14.4)$ & $28(13)$ & $259(15.6)$ & $26(13.3)$ & $254(14.8)$ & $17(13.2)$ & $261(14.8)$ \\
\hline$P$-value & $<0.003$ & & $<0.002$ & & $<0.033$ & & 0.031 & \\
\hline
\end{tabular}

Abbreviation: 25(OH)D, serum 25-hydroxyvitamin $\mathrm{D}$.

significantly lower compared with women without risk factors. Even multivariate logistic regression analysis supported this finding that vitamin D deficiency was a significant contributor for GDM (OR 1.387, $P=0.019)$ and anemia (OR 1.87, $P \leq 0.001)$.

Table 4 Maternal and obstetric characteristics of pregnant women with complications: gestational diabetes mellitus (GDM), anemia, iron deficiency, and preeclampsia $(n=1,873)$

\begin{tabular}{|c|c|c|c|c|}
\hline Variable & $\begin{array}{l}\text { GDM } \\
(n=260), \\
n(\%)\end{array}$ & $\begin{array}{l}\text { Anemia } \\
(n=216), \\
n(\%)\end{array}$ & $\begin{array}{l}\text { Iron } \\
\text { deficiency } \\
(n=162), \\
n(\%)\end{array}$ & $\begin{array}{l}\text { Preeclampsia } \\
(n=129), \\
n(\%)\end{array}$ \\
\hline \multicolumn{5}{|c|}{ Body mass index $\left(\mathrm{kg} / \mathrm{m}^{2}\right)$} \\
\hline Normal & $95(36.5)^{*}$ & $85(39.4)$ & $53(32.7)$ & $37(28.7)$ \\
\hline Overweight & $57(21.9)$ & $62(28.7)$ & $52(32.1)$ & $39(30.2)$ \\
\hline Obese & $108(41.5)^{*}$ & $69(31.9)^{*}$ & $57(35.2)^{*}$ & $53(4 I .1)^{*}$ \\
\hline \multicolumn{5}{|c|}{ Parity group } \\
\hline$<2$ & $64(24.6)$ & $66(30.6)^{*}$ & $35(21.6)$ & $31(24)$ \\
\hline $2-3$ & $72(27.7)$ & $43(19.9)$ & $50(30.9)$ & $39(30.2)$ \\
\hline $4-6$ & $103(39.6)$ & $99(45.8)$ & $65(40.1)$ & $53(4 I . I)$ \\
\hline$>6$ & $21(8.1)$ & $8(3.7)$ & $12(7.4)$ & $6(4.7)$ \\
\hline \multicolumn{5}{|c|}{ Previous abortion } \\
\hline Yes & $65(25.0)^{*}$ & $48(22.2)^{*}$ & $28(17.3)$ & $31(24.0)$ \\
\hline No & $195(75.0)$ & $168(77.8)$ & $134(82.7)$ & $98(76.0)$ \\
\hline \multicolumn{5}{|c|}{ Previous stillbirth } \\
\hline Yes & $29(11.2)$ & $20(9.3)$ & $12(7.4)$ & $12(9.3)$ \\
\hline No & $23 \mid(88.8)$ & $196(90.7)$ & $150(92.6)$ & I I (90.7) \\
\hline \multicolumn{5}{|c|}{ Antenatal care } \\
\hline Yes & $205(78.8)$ & $185(85.6)^{*}$ & $140(86.4)^{*}$ & $90(69.8)^{*}$ \\
\hline No & $55(21.2)$ & $31(14.4)$ & $22(13.6)$ & $39(30.2)$ \\
\hline \multicolumn{5}{|c|}{ Gestational group } \\
\hline $28-31$ weeks & $2(0.8)$ & $2(0.9)$ & $5(3.1)$ & $\mathrm{I}(0.8)$ \\
\hline $32-35$ weeks & $7(2.7)$ & $9(4.2)$ & $10(6.2)$ & $6(4.7)$ \\
\hline Above & $251(96.5)$ & $205(94.9)$ & $147(90.7)$ & $122(94.6)$ \\
\hline \multicolumn{5}{|l|}{36 weeks } \\
\hline \multicolumn{5}{|c|}{ Premature ruptured membrane } \\
\hline Yes & $35(13.5)^{*}$ & $10(4.6)$ & $9(5.6)$ & $9(7.0)$ \\
\hline No & $225(86.5)$ & $206(95.4)$ & $153(94.4)$ & $120(93.0)$ \\
\hline \multicolumn{5}{|c|}{ History of preterm delivery } \\
\hline Yes & $44(16.9)^{*}$ & $23(10.6)$ & $21(13.05)^{*}$ & $8(6.2)$ \\
\hline No & $216(83.1)$ & $193(89.4)$ & |4| (87.0) & $121(93.8)$ \\
\hline
\end{tabular}

Note: $* P<0.05$ when compared with uncomplicated group.
Gestational diabetes is a result of pregnancy-induced insulin resistance and impaired compensating insulin secretion. Vitamin D improves insulin sensitivity by enhancing insulin responsiveness to glucose transport. In a meta-analysis review of various studies, Clifton-Bligh and McElduff ${ }^{23}$ reported that pregnant women with GDM had significantly lower $25(\mathrm{OH}) \mathrm{D}$ levels than the comparison group. In our study sample, severe vitamin deficiency

Table 5 Predictors for gestational diabetes mellitus, anemia, iron deficiency, and preeclampsia in studied pregnant women using multivariate logistic regression $(n=I, 873)$

\begin{tabular}{|c|c|c|c|}
\hline Independent variable & $\begin{array}{l}\text { Odds } \\
\text { ratio }\end{array}$ & $\begin{array}{l}95 \% \text { confidence } \\
\text { interval }\end{array}$ & $P$-value \\
\hline \multicolumn{4}{|c|}{ Gestational diabetes mellitus } \\
\hline $\begin{array}{l}\text { Premature ruptured } \\
\text { membrane }\end{array}$ & 3.2 & $(2.0-5.0)$ & $\leq 0.001$ \\
\hline Previous low birth weight & 1.8 & $(1.2-2.6)$ & $\leq 0.001$ \\
\hline Previous stillbirth & 1.5 & $(0.98-2.4)$ & 0.06 \\
\hline Previous abortion & 1.4 & $(1.0-2.0)$ & 0.03 \\
\hline Vitamin D deficiency & 1.4 & $(I . I-I .8)$ & 0.02 \\
\hline Consanguinity (first cousin) & 1.3 & $(1.0-1.8)$ & 0.03 \\
\hline \multicolumn{4}{|l|}{ Anemia } \\
\hline Vitamin D deficiency & 1.87 & $(1.3-2.6)$ & $\leq 0.001$ \\
\hline $\begin{array}{l}\text { Consanguinity } \\
\text { (second cousin) }\end{array}$ & 1.64 & $(1.2-2.2)$ & $\leq 0.001$ \\
\hline $\begin{array}{l}\text { Lack of vitamin D } \\
\text { supplements }\end{array}$ & 1.46 & $(1.0-2.1)$ & 0.04 \\
\hline Less antenatal care & 1.43 & $(0.95-2.2)$ & 0.09 \\
\hline Nationality & 1.33 & $(0.99-1.8)$ & 0.05 \\
\hline Age-group & 1.12 & $(0.98-1.3)$ & 0.09 \\
\hline \multicolumn{4}{|l|}{ Iron deficiency } \\
\hline Gestational weeks & 2.77 & $(1.78-4.4)$ & $\leq 0.001$ \\
\hline Previous low birth weight & 2.17 & $(1.4-3.3)$ & $\leq 0.001$ \\
\hline Less antenatal care & 1.93 & $(1.2-3.1)$ & 0.01 \\
\hline Vitamin D deficiency & 1.84 & $(1.1-3.3)$ & 0.02 \\
\hline Nationality & 1.52 & $(I . I-2.1)$ & 0.01 \\
\hline Occupation & 1.30 & $(1.0-1.6)$ & 0.02 \\
\hline Age-group & 1.23 & $(1.0-1.5)$ & 0.02 \\
\hline \multicolumn{4}{|l|}{ Preeclampsia } \\
\hline Vitamin D deficiency & 1.75 & $(1.2-2.6)$ & 0.01 \\
\hline
\end{tabular}


Table 6 Vitamin D status during pregnancy or at delivery: global variations and comparisons

\begin{tabular}{|c|c|c|c|c|c|}
\hline Study & Country & Sample size & Sun exposure/vitamin D intake & Mean, ng/mL & Mean, nmol/L \\
\hline Dawodu and Akinbi ${ }^{3}$ & $\begin{array}{l}\text { United Arab } \\
\text { Emirates/Arabs }\end{array}$ & 192 & $\begin{array}{l}\text { Lack of sun exposure/low vitamin D } \\
\text { intake }\end{array}$ & 8.2 & 20.5 \\
\hline Jiang et $\mathrm{al}^{6}$ & $\begin{array}{l}\text { People's Republic } \\
\text { of China/Chinese }\end{array}$ & 152 & $\begin{array}{l}\text { Low sun exposure/low vitamin D } \\
\text { intake }\end{array}$ & $\begin{array}{l}9.1 \\
12.7\end{array}$ & $\begin{array}{l}22.7 \\
31.8\end{array}$ \\
\hline Holmes et $\mathrm{al}^{7}$ & UK/Caucasian & 99 & Low supplementation & NK & NK \\
\hline Javaid et $\mathrm{al}^{9}$ & UK/Caucasian & 160 & $\begin{array}{l}\text { Low UV exposure/low rate of } \\
\text { vitamin D supplementation in } \\
\text { pregnancy }\end{array}$ & NK & NK \\
\hline Bowyer et $\mathrm{al}^{10}$ & Australia/mixed & 971 & $\begin{array}{l}\text { Low sun exposure/sunscreen } \\
\text { use/vitamin } D \text { intake }\end{array}$ & $21 *$ & $52.0 *$ \\
\hline Hamilton et al'" & USA/mixed & 559 & Low sun exposure & 21.7 & 54.3 \\
\hline Sahu et al ${ }^{12}$ & India/Indian & 139 & $\begin{array}{l}\text { Inadequate exposure/low } \\
\text { vitamin } \mathrm{D} / \mathrm{Ca} \text { intake }\end{array}$ & 12.7 & 31.8 \\
\hline Viljakainen et al ${ }^{16}$ & Finland/Caucasian & 124 & $\begin{array}{l}\text { Low UVB exposure/inadequate } \\
\text { vitamin } D \text { intake }\end{array}$ & 16.4 & 41.0 \\
\hline Bassir et $\mathrm{a}^{35}$ & Iran/Iranian & 50 & $\begin{array}{l}\text { Lack of sun exposure/low } \\
\text { vitamin } D \text { intake }\end{array}$ & 5.1 & 12.8 \\
\hline Newhook et a ${ }^{36}$ & Canada/Caucasian & 50 & $\begin{array}{l}\text { Low UV exposure/low vitamin D } \\
\text { supplementation }\end{array}$ & $20.7 / 24.4$ & $51.9 / 61.1$ \\
\hline Ginde et $\mathrm{al}^{37}$ & USA/mixed & 928 & $\begin{array}{l}\text { Low outdoor activity/low } \\
\text { vitamin } D \text { intake }\end{array}$ & 26 & 65.0 \\
\hline Zhang et $\mathrm{a}^{38}$ & $\begin{array}{l}\text { People's Republic } \\
\text { of China }\end{array}$ & 953 & $\begin{array}{l}\text { Low sun exposure/low } \\
\text { vitamin } D \text { intake }\end{array}$ & $24.2 / 31$ & 24.2 \\
\hline Parlea et $\mathrm{a}^{39}$ & Canada & 116 & $\begin{array}{l}\text { Low UV exposure/low } \\
\text { vitamin D supplementation }\end{array}$ & 24 & 24 \\
\hline Merewood et a $\left.\right|^{40}$ & USA & 459 & $\begin{array}{l}\text { Low outdoor activity/low } \\
\text { vitamin } D \text { intake }\end{array}$ & 24.8 & 24.8 \\
\hline Burris et $\mathrm{al}^{41}$ & USA & $\mathrm{I}, 087$ & $\begin{array}{l}\text { Low outdoor activity/low } \\
\text { vitamin } D \text { intake }\end{array}$ & 23.6 & 59 \\
\hline Nicolaidou et $\mathrm{a}^{42}$ & Greece & 123 & $\begin{array}{l}\text { Lack of sun exposure/low } \\
\text { vitamin } D \text { intake }\end{array}$ & $6.6-8.2$ & $16.4-20.4$ \\
\hline Halicioglu et $\mathrm{al}^{43}$ & Turkey & 258 & $\begin{array}{l}\text { Lack of sun exposure/low } \\
\text { vitamin } D \text { intake }\end{array}$ & 11.5 & 11.5 \\
\hline Molla et $\mathrm{a}^{44}$ & Kuwait & 128 & $\begin{array}{l}\text { Low UVB exposure/low } \\
\text { vitamin } \mathrm{D} / \mathrm{Ca} \text { intake }\end{array}$ & 13.3 & 33.3 \\
\hline $\begin{array}{l}\text { Bener et al } \\
\text { (present study) }\end{array}$ & Qatar & 767 & $\begin{array}{l}\text { Lack of sun exposure/low } \\
\text { vitamin } D \text { intake }\end{array}$ & 17.8 & 44.5 \\
\hline
\end{tabular}

Notes: *Median; to convert serum 25 -hydroxyvitamin $\mathrm{D}$ to $\mathrm{ng} / \mathrm{mL}$, divide by 2.5 .

Abbreviations: NK, not known; UVB, ultraviolet B.

was significantly higher in pregnant women with GDM $(16.5 \%)$ than those without GDM (11\%), which is in line with findings from the cross-sectional study of Maghbooli et al. ${ }^{25}$ More than half of the complicated pregnant women with GDM (54.6\%) had vitamin deficiency. In the study of Zhang et al, ${ }^{26} 33 \%$ of GDM cases had vitamin $\mathrm{D}$ deficiency which was higher than in their comparable group (14\%). On the contrary, no significant association was observed between 25(OH)D concentrations and GDM risk in the Indian population. ${ }^{27}$ Another study from the $\mathrm{UK}^{28}$ failed to find a significant association between vitamin $\mathrm{D}$ deficiency and risk of GDM. These study findings highlight the possibility that vitamin D sufficiency may be important to maternal health, vitamin D sufficiency may be important to maternal health; vitamin $\mathrm{D}$ supplementation may be a simple way to reduce the risk of adverse pregnancy outcome.

Gestational anemia is common in developing countries, where it affects more than $57 \%$ of pregnancies. ${ }^{29}$ Our study is one of the first to investigate the association of vitamin D and anemia. The present study demonstrated a significantly greater risk of anemia in pregnant women with severe vitamin D deficiency (17.1\%) than that in normal groups (11\%). More than half of the pregnant women with anemia had vitamin D insufficiency (54.6\%), whereas it was lower in the normal group without anemia (47\%). Sim et $\mathrm{al}^{30}$ reported a significant correlation between vitamin D deficiency and anemia. Anemia is regarded as a major risk factor for maternal and perinatal mortality and morbidity. 
Similar to gestational diabetes and anemia, the data revealed a significant association between preeclampsia and severe vitamin D deficiency. Vitamin D deficiency was significantly higher in pregnant women with preeclampsia compared with the normal group (19.8\% vs $11.4 \%$ ), which is consistent with a meta-analysis ${ }^{31}$ showing a significant association between preeclampsia and 25(OH)D insufficiency. However, no significant association was observed in a prospective cohort study ${ }^{32}$ of pregnancies at high risk for preeclampsia in Canada. These study results revealed that maternal vitamin D deficiency was a strong, independent risk factor for preeclampsia. Vitamin D deficiency may also elevate blood pressure.

In the study sample, the risk of vitamin D deficiency was higher among young pregnant women below 30 years old (43.2\%), housewives $(65.3 \%)$, the educated $(41.1 \%)$, and those with low monthly household income $(<\mathrm{QR} 10,000)$ $(49.2 \%)$. In terms of lifestyle habits, vitamin D deficiency was higher among women with history of inadequate sun exposure (63.4\%) and less physical activity (64.4\%). It was reported ${ }^{32}$ that women with low socioeconomic status and inadequate sun exposure were more likely to have lower $25(\mathrm{OH}) \mathrm{D}$ concentrations than those of higher socioeconomic state and adequate sun exposure. In most countries, there is no monitoring of serum $25(\mathrm{OH}) \mathrm{D}_{3}$ levels during pregnancy for vitamin $\mathrm{D}$ deficiency. The vitamin D-deficiency epidemic during pregnancy is caused by a lack of adequate sunlight exposure needed to synthesize vitamin $\mathrm{D}_{3}$ in the skin, coupled with overall intakes that are too low to meet the increased demands of pregnancy. Previous studies of Bener et $\mathrm{al}^{33,34}$ support these findings that vitamin D deficiency was more prevalent in Qatar and the population had less exposure to sun, although it is a sunenriched population. Similar socioeconomic status of vitamin D deficiency was observed in pregnant women with GDM, anemia, and preeclampsia. Pregnant women who developed these maternal complications were younger, educated, housewives, and with low monthly household income. These findings provide evidence suggesting that vitamin D deficiency or insufficiency is common during pregnancy, which leads to adverse pregnancy outcome. Similar to the results observed in Qatar (17.8\%), vitamin D deficiency was higher in the US $(24.8 \%),{ }^{40}$ the People's Republic of China (24.2\%), ${ }^{38}$ Canada $(20.7 \%),{ }^{36}$ and Australia (21\%). ${ }^{10}$

The current study supports the hypothesis that vitamin D insufficiency is associated with an increased risk of such maternal complications as GDM, anemia, and preeclampsia. The vitamin D-deficiency epidemic during pregnancy is caused by a lack of adequate sunlight exposure needed to synthesis vitamin $\mathrm{D}_{3}$ in skin, coupled with overall intakes that are too low to meet the increased demand of pregnancy. Women living in the Middle East generally cover their body, so they receive little vitamin D from solar UVB. Also, it is so hot during the summer that people tend to stay indoors. The study findings suggest that low levels of $25(\mathrm{OH}) \mathrm{D}$ may be a modifiable risk factor in pregnancy, and health-care providers should at least be encouraging pregnant women to take vitamin $\mathrm{D}$.

\section{Conclusion}

The study findings revealed that maternal vitamin D deficiency in pregnancy is significantly associated with elevated risk for GDM, anemia, and preeclampsia. The risk of vitamin D deficiency was higher in Qataris, housewives, and those with low monthly house hold income. Vitamin D-deficient pregnant women had less exposure to sunlight, no physical activity, and less vitamin D supplement intake. Vitamin D supplementation in early pregnancy may be a simple way to reduce the risk of these adverse pregnancy outcomes.

\section{Acknowledgments}

This work was generously supported and funded by the Qatar Foundation, grant NPRP 08-760-3-153. The project was partially supported and funded by the Qatar Diabetic Association, Qatar Foundation. We also would like to thank Hamad Medical Corporation (10146/10) and the Weill Cornell Medical College Qatar Institutional Review Board (2010-0021) for their ethical approval of this study.

\section{Disclosure}

The authors report no conflicts of interest in this work.

\section{References}

1. Ndiweni Q, Buchmann EJ. Unbooked mothers and their babies - what causes the poor outcomes? SAfr Med J. 1998;88:192, 195-196, 199.

2. Harrison KA. Maternal mortality - a sharper focus on major issue of our time. Trop J Obstet Gynaecol. 1998;1:9-13.

3. Dawodu A, Akinbi H. Vitamin D nutrition in pregnancy: current opinion. Int $J$ Womens Health. 2013;5:333-343.

4. Mulligan ML, Felton SK, Riek AE, Bernal-Mizrachi C. Implications of vitamin D deficiency in pregnancy and lactation. Am J Obstet Gynecol. 2009;202:e429. e1-e9.

5. Wagner CL, Taylor SN, Dawodu A, Johnson DD, Hollis BW. Vitamin D and its role during pregnancy in attaining optimal health of mother and fetus. Nutrients. 2012;4:208-230.

6. Jiang L, Xu J, Pan S, Xie E, Hu Z, Shen H. High prevalence of hypovitaminosis D among pregnant women in southeast China. Acta Paediatr. 2012;101:e192-e194.

7. Holmes VA, Barnes MS, Alexander HD, McFaul P, Wallace JM. Vitamin D deficiency and insufficiency in pregnant women: a longitudinal study. Br J Nutr. 2009;102:876-881. 
8. Holick MF, Binkley NC, Bischoff-Ferrari HA, et al. Evaluation, treatment, and prevention of vitamin D deficiency: an Endocrine Society clinical practice guideline. J Clin Endocrinol Metab. 2011;96: 1911-1930.

9. Javaid MK, Crozier SR, Harvey NC, et al. Maternal vitamin D status during pregnancy and childhood bone mass at age 9 years: a longitudinal study. Lancet. 2006;367:36-43.

10. Bowyer L, Catling-Paull C, Diamond T, Homer C, Davis G, Craig ME. Vitamin D, PTH and calcium levels in pregnant women and their neonates. Clin Endocrinol (Oxf). 2009;70:372-377.

11. Hamilton SA, McNeil R, Hollis BW, et al. Profound vitamin D deficiency in a diverse group of women during pregnancy living in a sun-rich environment at latitude $32^{\circ} \mathrm{N}$. Int J Endocrinol. 2010;2010:917428.

12. Sahu M, Bhatia V, Aggarwal A, et al. Vitamin D deficiency in rural girls and pregnant women despite abundant sunshine in northern India. Clin Endocrinol (Oxf). May 2009;70:680-684.

13. Hollis BW, Wagner CL. Assessment of dietary vitamin $D$ requirements during pregnancy and lactation. Am J Clin Nutr. 2004;79:717-726.

14. World Health Organization. Worldwide Prevalence of Anaemia 1993-2005: WHO Global Database on Anaemia. Geneva: WHO; 2008.

15. Dawodu A, Wagner CL. Mother-child vitamin D deficiency: an international perspective. Arch Dis Child. 2007;92:737-740.

16. Viljakainen HT, Saarnio E, Hytinantti T, et al. Maternal vitamin D status determines bone variables in the newborn. $J$ Clin Endocrinol Metab. 2010;95:1749-1757.

17. Van der Meer IM, Karamali NS, Boeke AJ, et al. High prevalence of vitamin D deficiency in pregnant non-Western women in The Hague, The Netherlands. Am J Clin Nutr. 2006;84:350-353.

18. Vieth R, Bischoff-Ferrari H, Boucher BJ, et al. The urgent need to recommend an intake of vitamin $\mathrm{D}$ that is effective. Am J Clin Nutr. 2007;85:649-650.

19. Specker B. Vitamin D requirements during pregnancy. Am J Clin Nutr 2004;80:1740S-1747S.

20. [No authors listed]. World Health Organization-International Society of Hypertension Guidelines for the Management of Hypertension. Guidelines Subcommittee. J Hypertens. 1999;17:151-183.

21. Genuth S, Alberti KG, Bennett P, et al. Follow-up report on the diagnosis of diabetes mellitus. Diabetes Care. 2003;26:3160-3167.

22. Kaludjerovic J, Vieth R. Relationship between vitamin D during perinatal development and health. J Midwifery Womens Health. 2010;55: $550-560$.

23. Clifton-Bligh RJ, McElduff A. Maternal vitamin D deficiency, ethnicity and gestational diabetes. Diabet Med. 2008;25:678-684.

24. Cranney A, Horsley T, O'Donnell S, et al. Effectiveness and safety of vitamin D in relation to bone health. Evid Rep Technol Assess (Full Rep). 2007;158:1-23.

25. Maghbooli Z, Hossein-Nezhad A, Karimi F, Shafaei AR, Lanjani B. Correlation between vitamin D3 deficiency and insulin resistance in pregnancy. Diabetes Metab Res Rev. 2008;24:27-32.

26. Zhang C, Qiu C, Hu FB, et al. Maternal plasma 25-hydroxivitamin D concentrations and the risk for gestational diabetes mellitus. PLoS One. 2008;3:e3753.

27. Farrant HJ, Krishnaveni GV, Hill JC, et al. Vitamin D insufficiency is common in Indian mothers but is not associated with gestational diabetes or variation in newborn size. Eur J Clin Nutr. 2009;63:646-652.
28. Makgoba M, Nelson SM, Sarvidou M, Messow CM, Nicolaides K, Sattar N. First-trimester circulating 25-hydroxyvitamin D levels and development of gestational diabetes mellitus. Diabetes Care. 2011;34: 1091-1093.

29. McLean E, Cogswell M, Egli I, Wojdyla D, DeBenoist B. Worldwide prevalence of anaemia, WHO Vitamin and Mineral Nutrition Information System, 1993-2005. Public Health Nutr. 2009;12:444-454.

30. Sim JJ, Lac PT, Mcguerditchian SO, Kumar VA, Kujubu DA, Rasgon SA. Vitamin D deficiency and anemia: a cross-sectional study. Ann Hematol. 2010;89:447-452.

31. Robinson CJ, Alanis MC, Wagner CL, Hollis BW, Johnson DD. Plasma 25-hydroxyvitamin D levels in early-onset severe preeclampsia. Am J Obstet Gynecol. 2010;203:366. e1-e6.

32. Shand AW, Nassar N, Von Dadelszen P, Innis SM, Green TJ. Maternal vitamin D status in pregnancy and adverse pregnancy outcomes in a group at high risk for pre-eclampsia. BJOG. 2010;117:1593-1598.

33. Bener A, Alsaied A, Al-Ali M, et al. Impact of lifestyle and dietary habits on hypovitaminosis $\mathrm{D}$ in type 1 diabetes mellitus and healthy children from Qatar, a sun-rich country. Ann Nutr Metab. 2008;53:215-222.

34. Bener A, Al-Ali M, Hoffmann GF. High prevalence of vitamin D deficiency in young children in a highly sunny humid country: a global health problem. Minerva Pediatr. 2009;61:15-22.

35. Bassir M, Laborie S, Lapillonne A, Claris O, Chappuis MC, Salle BL. Vitamin D deficiency in Iranian mothers and their neonates: a pilot study. Acta Paediatr. 2001;90:577-579.

36. Newhook LA, Sloka S, Grant M, Randell E, Kovacs CS, Twells LK. Vitamin D insufficiency common in newborns, children and pregnant women living in Newfoundland and Labrador, Canada. Matern Child Nutr. 2009;5:186-191.

37. Ginde AA, Sullivan AF, Mansbach JM, Camargo CA Jr. Vitamin D insufficiency in pregnant and nonpregnant women of childbearing age in the United States. Am J Obstet Gynecol. 2010;202:436. e1-e8.

38. Lu HK, Zhang Z, Ke YH, He JW, Fu WZ, Zhang CQ, Zhang ZL. High prevalence of vitamin $D$ insufficiency in China: relationship with the levels of parathyroid hormone and markers of bone turnover. PLoS One. 2012;7(11):e47264.

39. Parlea L, Bromberg IL, Feig DS, Vieth R, Merman E, Lipscombe LL. Association between serum 25-hydroxyvitamin $\mathrm{D}$ in early pregnancy and risk of gestational diabetes mellitus. Diabet Med. 2012;29:e25-e32.

40. Merewood A, Mehta SD, Grossman X, et al. Widespread vitamin D deficiency in urban Massachusetts newborns and their mothers. Pediatrics. 2010;125:640-647.

41. Burris HH, Rifas-Shiman SL, Camargo CA Jr, et al. Plasma 25-hydroxyvitamin D during pregnancy and small-for-gestational age in black and white infants. Ann Epidemiol. 2012;22:581-586.

42. Nicolaidou P, Hatzistamatiou Z, Papadopoulou A, et al. Low vitamin D status in mother-newborn pairs in Greece. Calcif Tissue Int. 2006;78: 337-342.

43. Halicioglu O, Aksit S, Koc F, et al. Vitamin D deficiency in pregnant women and their neonates in spring time in western Turkey. Paediatr Perinat Epidemiol. 2012;26:53-60.

44. Molla AM, Al Badawi M, Hammoud MS, et al. Vitamin D status of mothers and their neonates in Kuwait. Pediatr Int. 2005;47: $649-652$.
International Journal of Women's Health

\section{Publish your work in this journal}

The International Journal of Women's Health is an international, peerreviewed open-access journal publishing original research, reports, editorials, reviews and commentaries on all aspects of women's healthcare including gynecology, obstetrics, and breast cancer. The manuscript management system is completely online and includes

\section{Dovepress}

a very quick and fair peer-review system, which is all easy to use. Visit http://www.dovepress.com/testimonials.php to read real quotes from published authors. 